\title{
La relación entre las prohibiciones del abuso de posición dominante y de las restricciones verticales
}

\author{
Carlos Noda Yamada*
}

\section{Objeto de estudio}

El objeto del presente comentario es abordar la problemática de la relación entre la prohibición del abuso de posición dominante en el mercado — contemplada en los artículos 3, 4 y 5 del decreto legislativo 701-y la prohibición de los acuerdos verticales anticompetitivos - contemplada en los artículos 3 y 6 del decreto legislativo 701 -

Dentro del objeto de la presente exposición, por tanto, se encuentra la lectura y análisis del significado de ambas prohibiciones, así como la obtención de una interpretación respecto a sus alcances, vinculaciones entre sí y limitaciones, todo ello, a la luz de la finalidad del ordenamiento en materia de defensa de la competencia.

Cabe advertir, sin embargo, que el presente comentario de ninguna manera tiene como propósito agotar el tratamiento de esta problemática; por el contrario, busca plantear el inicio de un debate teórico acerca de ella. En tal sentido, a lo largo de este trabajo, se buscarán los elementos comunes a la prohibición de los acuerdos verticales restrictivos y del abuso de posición dominante en el mercado, a partir de la finalidad del ordenamiento de defensa de la competencia, la cual se identifica con el mantenimiento y preservación del orden de mercado competitivo.

\footnotetext{
Abogado egresado de la Pontificia Universidad Católica del Perú (PUCP), es profesor de Derecho de la Competencia en la Facultad de Derecho y de Protección al Consumidor en la maestría en Derecho de la Propiedad Intelectual y de la Competencia de la PUCP.
} 


\section{El mercado competitivo como instrumento para la maximización del bienestar de los consumidores}

Es necesario mencionar que, para objeto del presente estudio, se asume que el mercado constituye únicamente un mecanismo de asignación de recursos que, en teoría, debería brindar resultados tendientes a la maximización del bienestar de los consumidores. Un elemento central para el funcionamiento adecuado de dicho mecanismo de asignación de recursos es la competencia, la cual puede ser catalogada como un mecanismo natural de control que disciplina y gobierna la actuación de las empresas en el mercado. Esto último significa que la competencia no es un fin en sí misma sino que solamente constituye un instrumento para lograr el resultado consistente en la maximización del bienestar de los consumidores.

\subsection{Origen del mercado competitivo}

El origen del actual mercado competitivo, basado en el principio de competencia y en el ejercicio libre de la iniciativa privada, puede ser ubicado - al menos en lo que respecta a Europa continental y a los territorios que pertenecieron a la América española - en la última década del siglo XVIII. En efecto, la realidad del Antiguo Régimen - tal como se conoce al estado de cosas vigente antes de la Revolución Francesa y la consiguiente expansión del programa revolucionario por el continente europeo - se caracterizaba por la organización estamental de la sociedad, un sistema legal basado en el casuismo y el privilegio, y relaciones económicas con fuerte presencia de corporaciones.

En el mundo del Antiguo Régimen no regía el principio de la libre iniciativa privada sino, por el contrario, un sistema en el cual los privilegios otorgados por la Corona a los gremios y corporaciones sujetaba la iniciativa de los particulares a dichos privilegios. Del mismo modo, no podía imaginarse la vigencia del principio de competencia; por el contrario, las relaciones económicas se encontraban fuertemente regladas por la Corona y, nuevamente, limitadas por los privilegios de las corporaciones.

La situación antes descrita cambió radicalmente como consecuencia de la Revolución Francesa y su consiguiente expansión por Europa continental, observándose la eliminación de los privilegios de las corporaciones — por ejemplo, a través de la implementación de las medidas de abolición de los privilegios por parte de la Asamblea Constituyente, en agosto de 1789_-, la supresión de los monopolios y la consagración de la libertad de comercio e industria - por ejemplo, mediante la Ley d'Allarde de marzo de 1791—y la prohibición de las corporaciones —mediante la Ley Le Chapelier de junio de 1791. 
Es claro que la implementación del programa revolucionario en el plano económico dio origen al actual orden de mercado competitivo, por lo que es a partir de aquel momento histórico que pueden empezar a rastrearse las razones que justifican defender dicho estado de cosas y, a la vez, puede estudiarse la evolución de los distintos ordenamientos relacionados con la defensa y preservación del referido orden de mercado.

\subsection{El mercado entendido como un sistema de balance de poder económico}

La noción de balance de poder es comúnmente utilizada por la teoría realista de las relaciones internacionales ${ }^{1}$ para describir una situación en la cual, asumiendo que el poder ${ }^{2}$ existe $y$, además, existe para ser utilizado, ${ }^{3}$ las relaciones entre los Estados se encuentran determinadas por la contraposición de poderes entre Estados o bloques de Estados. ${ }^{4}$ En tal sentido, la búsqueda del poder y la seguridad es el elemento lógico dominante de la política internacional, no teniendo los Estados más alternativa que acumular los medios de violencia para asegurar su propia supervivencia. ${ }^{5}$ Ello significa que el equilibrio en el sistema internacional es producto de la tensión entre los distintos poderes que actúan en el escenario de la política internacional; paradójicamente, entonces, la búsqueda de la paz y la búsqueda del poder se encuentran indisolublemente ligadas, bajo dicha perspectiva.

Al observar con cierto detenimiento el fenómeno económico del mercado, se puede apreciar la naturalidad con la que la noción de balance de poder es plenamente aplicable para explicar las relaciones que se dan al interior de dicho mecanismo de asignación de recursos. En efecto, el mercado, en una definición bastante básica y simplificada — pero suficiente para cumplir con el

\footnotetext{
La aproximación clásica al realismo en la disciplina de las Relaciones Internacionales debe hacerse a través de la lectura de la obra de Morgenthau. Ver Morgenthau, Hans J. Politics among nations. The struggle for power and peace. Séptima edición revisada por Kenneth W. Thompson y W. David Clinton. Nueva York: Mc Graw-Hill, 2006.

2 Poder entendido como el control de un ser humano sobre los pensamientos y las acciones de otros. A decir de esta teoría, el poder político es una relación psicológica entre quienes detentan el poder y aquellos sobre quienes se ejerce este. Ver MorGentHau, Hans J., ob. cit., p. 30.

3 De acuerdo con esta teoría, una potencia siempre busca una de las siguientes alternativas: (i) mantener el poder con el que cuenta; (ii) incrementar su poder; o (iii) demostrar su poder. Ibíd., p.50

4 Un ejemplo de esta situación es la que describe Kissinger refiriéndose al período inmediatamente posterior a la derrota definitiva de Napoleón en 1815. Ver KISSINGER, Henry. Diplomacy. Nueva York: Touchstone, 1995, p. 77.

5 BURCHILL, Scott. «Realism and Neo-realism». En BURCHILL, Scott y LINKLATER Andrew. Theories of international relations. Nueva York: St. Martin's Press, 1996. p. 81.
} 
objeto del presente estudio-, no es más que la confluencia, en el tiempo y en el espacio, de las denominadas fuerzas de la oferta y de la demanda. Bajo esta definición, la oferta representaría los intereses ${ }^{6}$ y la actuación de las empresas, mientras que la demanda expresaría los intereses y la actuación de los consumidores.

En el modelo de competencia perfecta —el cual no es más que una abstracción simplificada de la compleja realidad, pero muy útil para fines explicativos-, la oferta y la demanda se neutralizarían mutuamente, alcanzándose el equilibrio competitivo, el cual, por definición, constituye un equilibrio en tensión, producto de la contraposición —utilizando nuevamente términos realistas - de dos poderes, en este caso el poder de las empresas, de un lado, y el poder de los consumidores, del otro.

El balance de poder antes descrito es posible debido a que uno de los presupuestos del modelo de competencia perfecta es la presencia de un gran número de empresas y de consumidores, de tal modo que puede afirmarse que el mercado se encuentra atomizado. La consecuencia de dicha atomización es la falta de capacidad —o de poder - de cada empresa o consumidor — individualmente considerados - para ejercer influencia decisiva sobre los precios y la cantidad. Es evidente que, de no cumplirse el referido presupuesto del modelo - por ejemplo, debido a la formación de un cártel de empresas-, el resultado no sería el esperado balance de poder previsto por el modelo sino uno radicalmente distinto: el desequilibrio de poder.

\subsection{La defensa de la competencia como garantía del balance de poder económico}

En los mercados domésticos —entendiéndose como tales, los mercados nacionales o, en algunos casos, los mercados comunitarios-, el reconocimiento de que los presupuestos del modelo de competencia perfecta no se cumplen en la realidad, ha justificado la aparición de ordenamientos de defensa de la competencia. La finalidad de dichos ordenamientos es garantizar que el mercado cumpla su función de maximizar el bienestar de los consumidores, mediante la persecución y eliminación de aquellas conductas que tengan por objeto o efecto restringir la competencia. En términos de Bork:

- The only legitimate goal of American antitrust law is the maximization of consumer welfare; therefore,

6 De acuerdo con la teoría realista, el concepto de interés es definido en términos de poder. Ver Morgenthau, Hans J., ob. cit., p. 5. 
- 'Competition', for purposes of antitrust analysis, must be understood as a term of art signifying any state of affairs in which consumer welfare cannot be increased by judicial decree. ${ }^{7}$

La preservación del orden de mercado mediante los ordenamientos de defensa de la competencia se realiza garantizando la vigencia del mecanismo natural de control el cual las empresas se disciplinan mutuamente - la competencia-, impidiendo el ejercicio abusivo del poder por parte de las empresas, ya sea artificialmente conseguido — por medio de un cártel, por ejemplo- o producto de una situación natural —en una situación donde existe posición dominante individual o colectiva-; en ambos casos, dicho ejercicio abusivo del poder puede manifestarse excluyendo a empresas competidoras o explotando directamente a los consumidores.

En este punto de la exposición, nuevamente es útil acudir a los postulados de la teoría realista: el poder existe y, además, existe para ser utilizado; en tal sentido, esta utilización puede manifestarse en la pura y simple imposición sobre otros actores o, dado que el poder solamente puede ser contrarrestado por otro poder — de ahí la idea del balance de poder —, puede significar la obstaculización del surgimiento de poderes desafiantes y la concentración de mayor poder para sí mismo. Retornando al plano económico, la primera utilización equivale a una conducta de explotación directa mientras que la segunda corresponde a una conducta de exclusión de agentes rivales.

\section{El mercado competitivo como objeto de protección del ordenamiento en materia de defensa de la competencia}

La política y la legislación en materia de defensa de la competencia, a nivel mundial, han experimentado una gran evolución a lo largo de la historia, desde su aparición visible ${ }^{8}$ con la aprobación de la Sherman Act — una ley pionera de defensa de la competencia- en los Estados Unidos de América, ocurrida en 1890, hasta nuestros días. En efecto, el proceso histórico de la segunda mitad del siglo XIX y de todo el siglo XX, explican la influencia de la legislación norteamericana en materia de defensa de la competencia.

BORK, Robert H. The antitrust paradox. A policy at war with itself. Nueva York: Free Press, 1978-1993, p. 51.

8 Si bien la ley de defensa de la competencia canadiense —An Act for the Prevention and Suppression of Combinations Formed in Restraint of Trade (1889) — es anterior a la Sherman Act, esta última constituye uno de los instrumentos legislativos más conocidos e influyentes en materia de defensa de la competencia a nivel internacional. Para mayor información relacionada con la política y legislación de competencia canadiense, ver, por ejemplo, http:// www.internationalcompetitionnetwork.org/questionnaire_canada.pdf\#search='competition $\%$ 20authority\%20canada 
$\mathrm{Al}$ respecto, cabe recordar que, para el momento de la aprobación de la Sherman Act, los Estados Unidos de América ya habían superado la división que los condujo a la Guerra de Secesión (1861-1865), encontrándose en pleno proceso de crecimiento y expansión tanto económica como política. Es más, incluso antes de la guerra civil, los Estados Unidos habían demostrado su poder en el continente americano al derrotar a México en la guerra de 1847 y, hacia fines del siglo XIX, al derrotar a España y liquidar los restos de la monarquía universal —en 1898-, demostrando sus serias aspiraciones a convertirse en una potencia de primer orden a nivel mundial. Dichas aspiraciones se concretaron con la intervención estadounidense de 1917-1918 en la Primera Guerra Mundial. El ascenso de los Estados Unidos a la categoría de gran potencia se confirmó durante la Segunda Guerra Mundial y, al finalizar esta, se consolidó como una de las dos superpotencias del orden bipolar propio de la Guerra Fría, junto con la extinta Unión de Repúblicas Socialistas Soviéticas.

Es en este marco donde se produce la recepción de la política y legislación de defensa de la competencia en Europa Occidental —así como en el Japón, con la Ley Antimonopolios de 1947, ' también por influencia estadounidense-, que se materializará no solo en la aprobación de normas específicas de defensa de la competencia sino también en la inclusión de los principios de defensa del orden de mercado en los distintos ordenamientos de represión de la competencia desleal.

En efecto, tras el fin de la Segunda Guerra Mundial, la influencia estadounidense en Europa Occidental —en el marco del proceso histórico de la Guerra Fría- determinó no solamente la adopción de legislación en materia de defensa de la competencia en suelo europeo sino, además, la transición del modelo profesional al modelo social de represión de la competencia desleal, a través del entendimiento de la necesidad de la protección del orden de mercado como mecanismo de garantía del interés de los consumidores y del bienestar de la sociedad en general —objetivos propios de la legislación en materia de defensa de la competencia- ${ }^{10}$

El devenir histórico ha presenciado la especialización y perfeccionamiento de las técnicas de represión de las conductas anticompetitivas en general y, en particular, en el entendimiento de los efectos de los acuerdos verticales en el

Mayor información sobre el caso particular de Japón, puede encontrarse en http:// www.jftc.go.jp/e-page/index.html

10 Actualmente, el ilícito propio de la legislación de represión de la competencia desleal es definido como todo comportamiento que resulte objetivamente contrario a la buena fe, que tenga por objeto o efecto afectar el normal desenvolvimiento de las actividades económicas en el mercado. Al respecto, ver, Font Galán, Juan Ignacio y Luis María Miranda Serrano. Competencia desleal y antitrust. Sistema de ilícitos. Madrid: Marcial Pons, Ediciones Jurídicas y Sociales, 2005, pp. 17-25. 
mantenimiento de la competencia y, derivado de dicho entendimiento, en la redefinición de los alcances de la noción y prohibición de los acuerdos verticales restrictivos. ${ }^{11}$

Antes de proseguir con el discurso relacionado específicamente con los acuerdos verticales restrictivos y su relación con el abuso de posición dominante, como conductas anticompetitivas propiamente reconocidas y tipificadas por el ordenamiento en materia de defensa de la competencia, es imprescindible advertir la necesidad de reconocer la armonía de todo el sistema normativo garantizador del principio de competencia en el mercado, observándose una relación de complementariedad entre las disciplinas gemelas de la defensa de la competencia y de la represión de la competencia desleal. En efecto, la doctrina reconoce que «[...] cometido y función de la lealtad es ya, también, evitar $e l$ falseamiento de la libertad de competencia en el mercado» ${ }^{12}$ y que:

En rigor, son cuatro las disciplinas en las que se clasifica legislativamente el Derecho de la competencia: la de la competencia desleal, la de la defensa de la competencia, la de la publicidad comercial y la del comercio interior. Desde un punto de vista disciplinar, no parece, sin embargo, incorrecto dividirlo o clasificarlo sólo en dos - como hacemos en el texto-: la de la competencia desleal y la antitrust. La razón es fácil de entender: según una opinión muy generalizada, las disciplinas de la publicidad comercial y del comercio interior se integran, en realidad, en la disciplina general de la deslealtad concurrencial [...] Por eso se dice que la Ley de Competencia Desleal (LCD) no es la única fuente del Derecho de la competencia desleal en el Ordenamiento español; junto a ella se citan fundamentalmente [...] la Ley General de Publicidad de 11 de noviembre de 1988 (LGP), que regula la publicidad desleal, esto es, las deslealtades concurrenciales puestas en práctica a través de la publicidad comercial $[. ..]{ }^{13}$

La alusión a la relación de complementariedad entre las disciplinas que conforman el sistema normativo garantizador del principio de competencia en el mercado - defensa de la competencia y represión de la competencia desleal - no es ociosa ni carece de sentido, puesto que el reconocimiento de dicha relación sirve para lograr observar la plenitud de los objetivos perseguidos por el sistema.

En efecto, el ordenamiento en materia de defensa de la competencia busca que prevalezca el principio de competencia en el mercado, a fin de fomentar la eficiencia económica e incrementar el bienestar de los consumidores. Para ello, define el ilícito como la realización de cualquier conducta —individual

\footnotetext{
11 Cabe advertir que existe un sector de la doctrina contrario a dicha prohibición. Por ejemplo, ver, Bork, Robert H., ob.cit., pp. 291-298.

12 Font Galán, Juan Ignacio y Luis María Miranda Serrano, ob. cit., p. 26.

13 Ibíd., p.11 (nota 4).
} 
o colectiva - que pueda afectar el intercambio de bienes y servicios y que tenga por objeto o efecto restringir la competencia. El objeto de protección y la definición del ilícito por parte del ordenamiento en materia de represión de la competencia desleal buscan también, en último término, que prevalezca el principio de competencia en el mercado. Tal como señala la doctrina española:

[...] el ilícito desleal es hoy el ilícito general del sistema jurídico del Derecho de la competencia, como general es también la Ley que lo regula; el ilícito antitrust, sin embargo, es un ilicito especial, al igual que su Ley reguladora, también especial. Ambos ilícitos tienen el denominador común de sancionar y reprimir conductas concurrenciales abusivas del derecho de libertad de empresa y libertad de competencia, pero tales conductas sólo pueden ser reprimidas como antitrust (ex LDC) cuando producen un impacto de mercado lesivo para los intereses públicos; razón por la cual la ilicitud antitrust es, en rigor, una deslealtad cualificada, esto es, una deslealtad que incide negativamente de modo relevante sobre los intereses generales, cuyo control se confiere por tal razón a la Administración Pública. ${ }^{14}$

Es claro que el ordenamiento en materia de defensa de la competencia - es más, de la totalidad del sistema normativo garantizador del principio de competencia en el mercado, incluyendo al ordenamiento en materia de represión de la competencia desleal—, tiene por finalidad última la promoción y el incremento del interés económico general —incluyendo al interés de los consumidores_-, mediante la preservación del orden económico de mercado —obviamente competitivo- , considerado como el mecanismo para lograr el mayor bienestar de la sociedad por el marco constitucional. Es a partir de dicha finalidad que debemos indagar acerca de los alcances y limitaciones de la relación entre las prohibiciones de los acuerdos verticales anticompetitivos y del abuso de posición dominante en el mercado.

\section{La relación entre la prohibición del abuso de posición dominante y la prohibición de los acuerdos verticales anticompetitivos}

Una aproximación hacia la relación entre la prohibición de los acuerdos verticales anticompetitivos y la prohibición del abuso de posición dominante debe necesariamente hacer referencia a la regulación contenida en el decreto legislativo 701 , respecto a dichas prohibiciones.

14 Ibíd., p. 36. 
$\mathrm{Al}$ respecto, la lectura conjunta de lo dispuesto por los artículos $3^{15}$ y $6^{16} \mathrm{del}$ decreto legislativo 701 permite observar que el contenido de la prohibición de las prácticas colusorias alcanza a todos aquellos acuerdos entre empresas, que produzcan o puedan producir el efecto de restringir, impedir o falsear la competencia, generando perjuicio para el interés económico general. Cabe aclarar que el término acuerdo, en el sentido utilizado por el ordenamiento nacional, no se refiere exclusivamente a los contratos como fuente de las obligaciones sino más bien a aquel género de actos en los que una de las partes se obliga a limitar su libertad de acción respecto a la otra, con la finalidad última de restringir la competencia. ${ }^{17}$

Los acuerdos verticales comprendidos dentro de los alcances de la prohibición de los artículos 3 y 6 del decreto legislativo 701 son aquellos actos celebrados por un productor con un distribuidor independiente que adquiere las mercancías y las revende - ya sea a minoristas o a consumidores finales-, asumiendo el riesgo de la transacción, ${ }^{18}$ de tal modo que produzcan o puedan producir el efecto de restringir, impedir o falsear la competencia, generando perjuicio para el interés económico general. Es evidente que no todos los acuerdos verticales -en sentido amplio, acuerdos celebrados entre sujetos que se encuentran en niveles distintos de la cadena de distribución - se encuentran dentro del ámbito de la prohibición sino, por el contrario, únicamente aquellos que sean capaces de producir efectos anticompetitivos y perjuicio al interés económico general.

Respecto de la capacidad de los acuerdos verticales de producir efectos anticompetitivos y, por tanto, perjudicar el interés económico general, se puede simplificar el panorama — por razones de claridad expositiva—, reduciéndolo a dos posiciones diametralmente opuestas. La primera enjuiciaría negativamente a los acuerdos verticales, enfatizando su carácter anticompetitivo, evidenciado — a decir de dicha postura - a través de la posibilidad, por ejemplo, de compartimentar

15 Decreto legislativo 701, artículo 3: «Actos y conductas prohibidas. Están prohibidos y serán sancionados, de conformidad con las normas de la presente Ley, los actos o conductas, relacionados con actividades económicas, que constituyen abuso de una posición de dominio en el mercado o que limiten, restrinjan o distorsionen la libre competencia, de modo que se generen perjuicios para el interés económico general, en el territorio nacional.

16 Decreto legislativo 701, artículo 6: «Prácticas restrictivas de la libre competencia. Se entiende por prácticas restrictivas de la libre competencia los acuerdos, decisiones, recomendaciones, actuaciones paralelas o prácticas concertadas entre empresas que produzcan o puedan producir el efecto de restringir, impedir o falsear la competencia. [...].

17 Bellamy, Christopher y Graham Child. Derecho de la competencia en el mercado común. Madrid: Civitas, 1992. p. 78.

18 Este hecho los diferenciaría de los acuerdos de agencia. Para mayor referencia acerca de la noción de acuerdos verticales, ver EChEVARria SÁENZ, Joseba Aitor. «Acuerdos verticales». En Velasco San Pedro, Luis Antonio (coordinador). Derecho europeo de la competencia (antitrust e intervenciones públicas). Valladolid: Lex Nova, 2005. p. 106. 
mercados, facilitar la cartelización, reducir la competencia intrabrand o elevar las barreras de acceso al mercado. La segunda visión correspondería a una lectura positiva respecto a los acuerdos verticales, señalando su inocuidad inicial, a menos que se verificara la existencia de una empresa en posición dominante en el mercado.

En relación con este tema, cabe recordar que en la doctrina comunitaria europea se han identificado tres posturas respecto de los acuerdos verticales que, en líneas generales, corresponden a los diversos períodos de evolución de la práctica comunitaria de enjuiciamiento a aquel tipo de acuerdos:

- La que entendía que eran acuerdos restrictivos per se, prohibidos por tanto, aunque susceptibles ocasionalmente de ser eximidos por la vía del artículo 81 , inciso 3 . El fundamento del artículo 81 sería conforme a esta visión, garantizar la independencia de las empresas y favorecer la existencia del mayor número posible de las mismas como fórmula de promoción de la libre competencia. Esta doctrina entiende que las empresas que forman parte del iter distributivo — productores, mayoristas y minoristascompiten tanto entre sí (competencia intrabrand) como con otros distribuidores de productos o servicios competidores (competencia interbrand). La política de la competencia tendría por ello como finalidad salvaguardar todos los niveles de competencia. [...].

- La que entendía que los acuerdos verticales introducían restricciones formales a la competencia, pero que dichas limitaciones sólo eran relevantes en función de sus efectos sobre el mercado («[...] que tengan por objeto o por efecto impedir, restringir o falsear la competencia en el Mercado Común»). Conforme a esta visión el objetivo del artículo 81 TUE es buscar una competencia eficaz o practicable (Workable competition) que garantice ciertos resultados al mercado y a los consumidores. No se trata, por tanto, de garantizar la independencia a ultranza de cada empresario, sino la existencia de un número suficiente de los mismos que permita al consumidor elegir. Por ello, frente a un acuerdo vertical no corresponde efectuar un análisis abstracto de sus cláusulas, sino que corresponde efectuar un análisis concreto de rule of reason o 'regla de la razón', que valorara: primero, si dichos acuerdos tienen un efecto anticompetitivo real o potencial y después, si dichas restricciones pueden verse compensadas por unos efectos más beneficiosos, acordes con lo establecido en el artículo 81, inciso 3 y, por lo tanto, declararse exentos. [...]. Conforme a esta doctrina resulta importante que el derecho de la competencia salvaguarde un nivel razonable de competencia intrabrand - entre el suministrador y los distribuidores minoristas, pero sobre todo entre estos últimos—, con el fin de garantizar las importaciones paralelas de productor a distribuidor o entre estos últimos y a través de las fronteras estatales, como mecanismo que asegura la permeabilidad y competitividad de los precios. La defensa 
de los consumidores, por tanto, se constituye también en un objetivo paritario con la defensa de la competencia en sí misma.

- La que entendía que los acuerdos verticales no restringen la competencia, siendo por el contrario un factor de incremento de la misma y que por tanto no estaban prohibidos por el artículo 81 del Tratado, a no ser que al disfrutar de una posición de dominio en el mercado, por sus efectos anticompetitivos, incurrieran en dicha prohibición, y aun entonces, sería necesario comprobar si concurrían o no las posibles causas de exención del artículo 81 inciso 3 del Tratado. Conforme a esta visión, los acuerdos verticales son lícitos per se, y sólo atendiendo a las circunstancias económicas y efectos del acuerdo podrían verse prohibidos. O más concretamente, los acuerdos verticales sólo serían un ilícito en conjunción con acuerdos horizontales o en mercados monopólicos u oligopólicos. Esta visión, propia del Derecho norteamericano, se manifiesta abiertamente antiintervencionista y discute la licitud del objetivo de tutela de la competencia intrabrand (sería un paternalismo innecesario), a la par que critica el error de principio que supone dar preeminencia a la tutela del consumidor frente a la incentivación de la competencia. ${ }^{19}$

De la cita anterior, puede observarse que la clasificación de posturas en la doctrina comunitaria, si bien indudablemente es más amplia que la empleada en el presente trabajo, no es contradictoria con esta sino complementaria a título informativo. Es evidente que, para mayor información y una mejor comprensión acerca del tratamiento comunitario actual de los acuerdos verticales, es necesario revisar el Reglamento (CE) 2790/1999 de la Comisión, del 22 de diciembre de 1999, así como la comunicación de la Comisión «Directrices relativas a las restricciones verticales» (2000/C 291/01).

Una interpretación equilibrada de la prohibición de los acuerdos verticales anticompetitivos requiere apartarse de las posturas maximalistas a fin de dar cabida a un análisis concreto — caso por caso-, que tome en consideración la figura contractual específica utilizada — ya se trate de un acuerdo de suministro exclusivo, de un contrato de distribución selectiva o de algún otro mecanismo análogo-y las particularidades de la industria en la que se manifiesta la conducta. Asimismo, debe tenerse presente la necesidad de analizar cuidadosamente no solo los efectos que el acuerdo pudiera tener sino también su objeto - o finalidad—, el cual, de ser exclusorio o, en general, anticompetitivo, determinaría la ilicitud del acuerdo.

La necesidad de una interpretación de la prohibición de los acuerdos verticales anticompetitivos que atienda a las particularidades concretas de cada caso - tanto por la figura contractual como por las condiciones de la industriase hace evidente si recordamos que toda forma de integración vertical — ya sea

19 Echevarria Sáenz, Joseba Aitor, ob.cit., pp. 108-110. 
por expansión interna o por concentración empresarial — o de contrato de exclusividad, tiene un efecto exclusorio sobre los agentes económicos rivales. De igual modo, si retrocedemos a una idea fundamental, no podemos dejar de reconocer que toda estrategia que se traduzca en un incremento de las preferencias de los consumidores por el establecimiento del empresario que implementa dicha estrategia, tiene un efecto exclusorio sobre los agentes económicos rivales. En otras palabras, en los supuestos mencionados -integración vertical, contratos de exclusividad y estrategias comerciales - se produce un daño concurrencial.

Este último es consustancial a la dinámica del mercado y, como tal, no puede ser juzgado como ilícito. Únicamente merecerá la calificación de ilícito cuando el referido daño o perjuicio sea producto de un acto anticompetitivo, ya sea, por ejemplo, porque la integración vertical o el contrato de exclusividad estaban diseñados especialmente para limitar la permanencia o impedir el acceso de competidores —objeto exclusorio y, por tanto, anticompetitivo- o para estabilizar y viabilizar un cártel —nuevamente, un objeto anticompetitivo-, o porque el efecto exclusorio es logrado por medio de un notable desperdicio de recursos dedicados a la tarea de monopolizar el mercado. ${ }^{20}$ En todos estos casos podemos observar la presencia de una conducta anticompetitiva que genera un perjuicio al interés económico general.

Lo anteriormente expuesto nos lleva a hacer referencia al contenido de la prohibición del abuso de posición dominante regulado en los artículos 3, $4^{21}$ y $5^{22}$ del decreto legislativo 701. La lectura conjunta de dichas disposiciones permite observar que el contenido de la prohibición del abuso de posición dominante alcanza a todas aquellas conductas desarrolladas por empresas dominantes, que produzcan o puedan producir el efecto de restringir, impedir o falsear la competencia, generando perjuicio para el interés económico general.

20 Para una interesante y útil referencia de la doctrina norteamericana respecto a este tema, ver Posner, Richard A. Antitrust Law. Segunda edición. Chicago y Londres: The University of Chicago Press, 2001, pp. 223-244.

21 Decreto legislativo 701, artículo 4: «Posición de dominio en el mercado. Se entiende que una o varias empresas gozan de una posición de dominio en el mercado, cuando pueden actuar de modo independiente con prescindencia de sus competidores, compradores, clientes o proveedores, debido a factores tales como la participación significativa de las empresas en los mercados respectivos, las características de la oferta y la demanda de los bienes o servicios, el desarrollo tecnológico o servicios involucrados, el acceso de competidores a fuentes de financiamiento y suministros, así como a redes de distribución.

22 Decreto Legislativo 701, artículo 5: «Abuso de posición de dominio en el mercado. Se considera que existe abuso de posición de dominio en el mercado, cuando una o más empresas que se encuentran en la situación descrita en el artículo anterior, actúan de manera indebida, con el fin de obtener beneficios y causar perjuicios a otros, que no hubieran sido posibles, de no existir la posición de dominio. [...]. 
Al igual que en el caso de la prohibición de los acuerdos verticales anticompetitivos, es evidente que no todas las conductas de una empresa dominante se encuentran dentro del ámbito de la prohibición sino, por el contrario, únicamente aquellas que sean capaces de producir efectos anticom-petitivos y perjuicio al interés económico general. En otras palabras, no es la posición dominante de la empresa la que es objeto de la prohibición, ni siquiera lo es el ejercicio de dicha posición dominante sino, más bien, el ejercicio abusivo de aquella, entendiendo por abusivo, a la capacidad de producción de efectos anticompetitivos y, por tanto, de perjudicar el interés económico general.

En este punto, una vez ensayada una aproximación al contenido de la prohibición de los acuerdos verticales anticompetitivos y del abuso de posición dominante, si volvemos a la noción de acuerdos verticales - como pura cuestión de hecho y sin ninguna carga valorativa-, encontraremos que la verificación en la realidad de un acto celebrado por un productor con un distribuidor independiente que adquiere las mercancías y las revende, asumiendo el riesgo de la transacción, puede tener significación material para el ordenamiento en materia de defensa de la competencia, en tanto dicho acto tenga la capacidad de producir efectos anticompetitivos, afectando el interés económico general.

Puede observarse que, en ambos casos, el contenido de la prohibición no atiende al instrumento o vehículo a través del cual se materializa la afectación anticompetitiva del interés económico general —en este caso, el acuerdo vertical— sino, más bien, atiende a la afectación en sí. Debido a ello, la conclusión obtenida para el caso de la ilicitud de los acuerdos verticales anticompetitivos sigue siendo válida, pudiendo extenderse a la ilicitud del abuso de posición dominante en contextos verticales, es decir, materializados en acuerdos verticales, de acuerdo a la definición anteriormente propuesta. En tal sentido, la prohibición atenderá, entre otras posibilidades, al hecho de que la conducta estaba diseñada especialmente para limitar la permanencia o impedir el acceso de competidores, para estabilizar y viabilizar un cártel, o porque el efecto exclusorio de la conducta es logrado por medio de un notable desperdicio de recursos dedicados a la tarea de monopolizar el mercado. En todos estos casos, el elemento común es la presencia de una conducta anticompetitiva que genera un perjuicio al interés económico general.

En vista de lo anterior, no puede afirmarse que los acuerdos verticales sean siempre anticompetitivos, o lo que es lo mismo, que se encuentren prohibidos per se: ello sería un despropósito puesto que significaría hacer recaer el contenido de la prohibición en un elemento de hecho, es decir, en el instrumento o vehículo de la posible afectación — la cual, de acuerdo a esta postura, ni siquiera debería ser probada- y no en la afectación en sí, negando, a la vez, los beneficios que dichos acuerdos pueden aparejar para un mayor grado de competencia en el mercado. En cuanto a esto último — los beneficios_-, basta recordar que los 
sistemas de distribución son un elemento central de toda estrategia integral de mercadotecnia.

No obstante, tampoco puede afirmarse que los acuerdos verticales sean siempre pro competitivos - $\mathrm{O}$ al menos neutrales desde el punto de vista de la competencia-, a menos que se verificara la existencia de una empresa en posición dominante en el mercado. Ello nuevamente significaría hacer recaer el contenido de la prohibición - esta vez, de la prohibición del abuso de posición dominante- en un elemento de hecho, es decir, en la situación de posición dominante de la empresa que posibilitaría la materialización de la afectación, y no en la afectación en sí.

En conclusión, la relevancia práctica de la distinción entre la prohibición de las prácticas colusorias y la prohibición del abuso de posición dominante, en lo que a los acuerdos verticales anticompetitivos se refiere, radicaría en la mayor probabilidad de encontrarnos ante una conducta materialmente capaz de restringir, falsear o limitar la competencia y que, por tanto, perjudique el interés económico general, en aquellos casos en que dicha conducta sea implementada por un agente en posición dominante. Esta posición parece ser consistente, en líneas generales, con la práctica y doctrina comunitaria:

The Commission's approach, stated expressly in the Guidelines, is that restrictions in distribution agreements are frequently economically beneficial as long as interbrand competition remains strong. Most restrictive clauses need to be looked at in their market and economic context in order to establish whether they do in fact constitute an appreciable restriction on competition. Relevant factors include market shares and market structure, product differentiation, barriers to entry, the existence of potential competition, collusion or oligopolistic behaviour between competing firms, buyer power, and the cumulative effects of a number of similar networks.

Restrictions on interbrand competition are normally viewed with more concern than restrictions on intrabrand competition. Where a supplier has a strong market presence then exclusive purchase and non-compete clauses will raise issues, because of the risk to interbrand competition where competing suppliers cannot find distributors for their products. ${ }^{23}$

Ello no significa afirmar que en todo caso en el que nos encontremos ante un agente en posición dominante, la conducta será anticompetitiva, y, de otro lado, tampoco significa negar la posibilidad de que, aun ante la inexistencia de un agente dominante, nos pudiéramos encontrar ante una conducta anticompetitiva.

23 Goyder, D.G. EC Competition Law. Cuarta edición. Oxford: Oxford University Press, 2003, p. 177. 


\section{Conclusión}

Como reflexión final, vale la pena recordar la finalidad del ordenamiento en materia de defensa de la competencia, la cual es la promoción y el incremento del interés económico general —incluyendo el interés de los consumidores-, mediante la preservación del orden económico de mercado, considerado como el mecanismo para lograr el mayor bienestar de la sociedad por el marco constitucional. En tal sentido, toda prohibición propia de dicho ordenamiento estará dirigida a obtener dicha finalidad, no siendo la prohibición de los acuerdos verticales anticompetitivos - como práctica colusoria o como abuso de posición dominante-, la excepción. Ello, por supuesto, sin negar la necesidad de una correcta y adecuada tipificación e imputación de cargos por parte de la autoridad instructora y de la exacta aplicación de una u otra prohibición, según el caso.

Precisamente, el reconocimiento de que el ordenamiento en materia de defensa de la competencia tiene por finalidad última la promoción y el incremento del interés económico general, mediante la preservación del orden económico de mercado, este último considerado como el mecanismo para lograr el mayor bienestar de la sociedad por el marco constitucional, hace que la interpretación más equilibrada de la prohibición de los acuerdos verticales anticompetitivos exija un análisis concreto — caso por caso-, que tome en consideración la figura contractual específica utilizada y las particularidades de la industria en la que se manifiesta la conducta presuntamente restrictiva.

Finalmente, dicho reconocimiento de la finalidad promotora del interés económico general hace que deba tenerse presente la necesidad de analizar cuidadosamente no solo los efectos que el acuerdo vertical pudiera tener sino también su objeto —o finalidad-, el cual, de ser exclusorio o, en general, anticompetitivo, determinaría su ilicitud. 